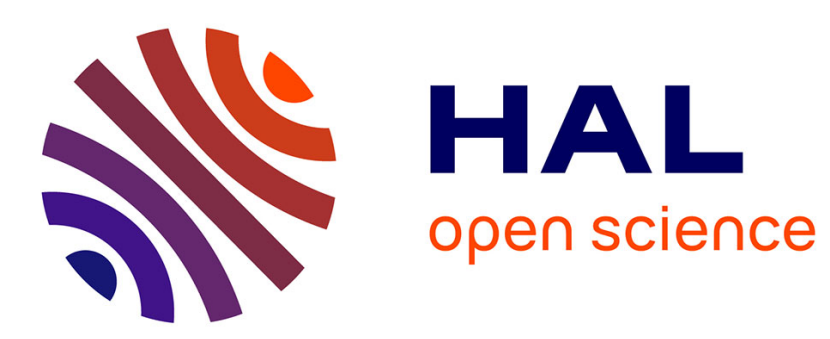

\title{
A Minimal Model of a Single-Reed Instrument Producing Quasi-Periodic Sounds
}

\author{
J.-B. Doc, C. Vergez, S. Missoum
}

\section{To cite this version:}

J.-B. Doc, C. Vergez, S. Missoum. A Minimal Model of a Single-Reed Instrument Producing Quasi-Periodic Sounds. Acta Acustica united with Acustica, 2014, 100 (3), pp.543-554(12). 10.3813/AAA.918734. hal-01087013

\section{HAL Id: hal-01087013 \\ https://hal.science/hal-01087013}

Submitted on 25 Nov 2014

HAL is a multi-disciplinary open access archive for the deposit and dissemination of scientific research documents, whether they are published or not. The documents may come from teaching and research institutions in France or abroad, or from public or private research centers.
L'archive ouverte pluridisciplinaire HAL, est destinée au dépôt et à la diffusion de documents scientifiques de niveau recherche, publiés ou non, émanant des établissements d'enseignement et de recherche français ou étrangers, des laboratoires publics ou privés. 


\title{
A minimal model of a single-reed instrument producing quasi-periodic sounds
}

\author{
J.-B. Doc ${ }^{1, a}$, C. Vergez ${ }^{1}$ and S. Missoum ${ }^{2}$ \\ ${ }^{1}$ LMA, CNRS, UPR 7051, Aix-Marseille Univ, Centrale Marseille, \\ F-13402 Marseille Cedex 20, France \\ ${ }^{2}$ Computational Optimal Design of Engineering Systems (CODES) Laboratory, \\ Department of Aerospace and Mechanical Engineering, \\ University of Arizona, Tucson, Arizona 85721
}

\begin{abstract}
Single-reed instruments can produce multiphonic sounds when they generate quasi-periodic oscillations. The aim of this article is to identify a minimal model of a single reedinstrument producing quasi-periodic oscillations. To better understand the influence of model parameters on the production of quasi-periodic regimes, the mapping between parameters and quasi-periodic regimes is explicitly identified using a support vector machine (SVM) classifier. SVMs enable the construction of boundaries between quasi-periodic and periodic regimes that are explicitly defined in terms of the parameters. Results and conclusions obtained from the numerical model are compared to published experiments related to the the production of quasi-periodic oscillations with an alto saxophone. This qualitative comparison highlights the influence of key parameters on the production of multiphonic sounds.
\end{abstract}

\section{List of symbols}

$\begin{array}{ll}\gamma & \text { Dimensionless blowing pressure } \\ \zeta & \text { Dimensionless reed opening parameter } \\ \sim & \text { Superscript representing physical quantities } \\ \omega_{n} & \text { Resonance pulsation of the } n^{t h} \text { mode of the resonator } \\ \mathcal{F}_{n} & \text { Resonance frequency of the } n^{t h} \text { mode of the resonator } \\ F_{n} & \text { Modal factor of the } n^{t h} \text { mode of the resonator } \\ Q_{n} & \text { Quality factor of the } n^{t h} \text { mode of the resonator } \\ f_{i} & \text { Remarkable frequency in a sound spectrum } \\ p & \text { Dimensionless mouthpiece pressure } \\ u & \text { Dimensionless air flow }\end{array}$

\section{Introduction}

Woodwind musical instruments are built using simple geometrical shapes (e.g., straight cylinder, cone). The manufacture of these instruments requires changes to the basic waveguide: lateral holes are drilled and a mouthpiece is connected (which requires to truncate the cone for saxophones). These modifications have the effect of altering the harmonicity between the resonance frequencies of the instrument. The inharmonicity of

\footnotetext{
${ }^{a}$ Corresponding authors: jbdoc@lma.cnrs-mrs.fr
} 
the resonance frequencies has a direct influence on the tuning of notes produced, especially when changing register [1]. Similarly, inharmonicity affects the timbre of the sounds produced by the instrument [2]. Inharmonicity is therefore a key aspect to consider in the design of musical instruments.

The single reed instruments can be considered as self-sustained oscillators. Through a valve effect, the mouthpiece ensures the conversion of a quasi-static pressure (inside the mouth) into an alternating pressure (inside the mouthpiece) which is sustained by the acoustic feedback of the air column filling the bore. The natural frequencies of the bore being inharmonic, the self-sustained oscillator can produce complex oscillation regimes. Among these, quasi-periodic oscillations have the particularity of being composed at least of two incommensurate frequencies [3]. Such regimes are a variety of multiphonic sounds [4]. The quasi-periodic oscillations in musical instruments are also encountered for the case of the wolf tone for the string instruments [5], and likewise in a study on the interaction between the resonance of the air column and the wall of an organ pipe [6].

Multiphonic sounds can be easily obtained with wind instruments if the instrument is played in an unconventional way. For example, for fork fingerings or non-standard embouchure adjustments, it is possible to obtain sounds perceived as rough or beating [7]. In this case, quasi-periodic regimes are sought by the musician to produce sound effects in contemporary music or jazz. However, even with a standard embouchure adjustment, quasiperiodicity may occur as an undesired event that instrument makers would like to avoid. The quasi-periodic regimes have been studied for organ pipes and flutes [8-10]. However, the quasi-periodic regimes produced by wind instruments $[11,12]$ are rarely studied and their emergence is not completely understood. It can be noted that the article [13] addresses the subject of quasi-periodic oscillations through an experimental study on tuning and intonation of reed instruments. In that paper, an artificial mouth is used with a saxophone to play the instrument in a controlled manner. The inharmonicity of the instrument is changed through pistons connected in parallel at the neck of the instrument. Quasi-periodic sounds are then obtained beyond a certain value of inharmonicity. Also, a particular adjustment of the embouchure is reported to favor the occurrence of quasi-periodic oscillations.

The purpose of the present article is to identify a minimal model of single reed-instrument derived from [14] producing quasi-periodic oscillations, and to check whether this model can qualitatively reproduce numerically behaviors observed experimentally in [13]. To better understand the influence of model parameters on the production of quasi-periodic regimes, the mapping between parameters and quasi-periodic regimes is explicitly identified using a technique referred to as Explicit Design Space Decomposition (EDSD) $[15,16]$ which is based on support vector machine classifiers (SVMs) $[17,18]$. SVMs enable the construction of boundaries between quasi-periodic and periodic regimes that are explicitly defined in terms of the parameters.

The paper is structured as follows. Section 2 introduces the single reed instrument model used in this work. In Section 3, the classification criterion to segregate oscillating regimes is presented. The EDSD approach is also introduced in that section. Finally, Section 4 compares the numerical predictions to experimental results already published in $[13]$. 


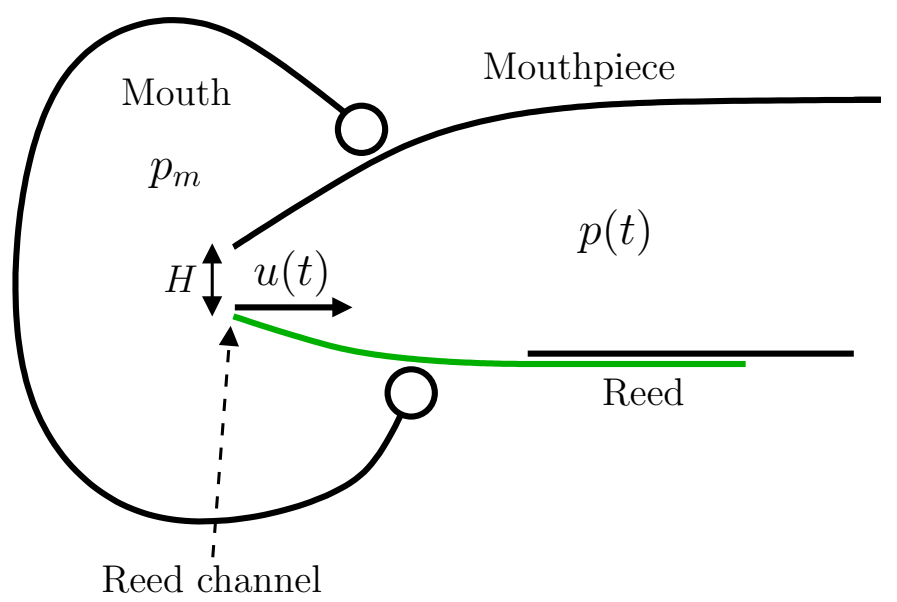

Fig. 1: Schematic representation of a musician playing a single-reed instrument.

\section{Classical single-reed instrument model}

The model described in this section is derived from [14,19] and it will be used in Section 3 to calculate solutions showing quasi-periodic oscillation.

\subsection{Continuous time equations}

By blowing air into the instrument through the reed channel, the player destabilizes the reed from its rest position (see Figure 1). The acoustic response of the instrument acts as a feedback loop which influences the reed position. The production of a sound corresponds to the self-sustained oscillation of this dynamical system. The reed can be modeled as a mass/spring/damper oscillator. However, because its resonance frequency is large compared to the first harmonics of typical playing frequencies, inertia and damping of the reed are ignored in this paper (the relevance of this approximation is discussed in Appendix A).

Considering as a reference the minimum pressure $p_{M}=K H$ required to close the reed channel in the non-oscillating case (where $K$ is the reed stiffness and $H$ is the height of the reed channel at rest), we introduce the following dimensionless quantities for the pressure in the mouth and in the mouthpiece as well as for the volume flow through the reed channel respectively (see Figure 1):

$$
\begin{aligned}
& \gamma=\frac{p_{m}}{p_{M}}, \\
& p(t)=\frac{\tilde{p}(t)}{p_{M}}, \\
& u(t)=\frac{Z_{c} \tilde{u}(t)}{p_{M}},
\end{aligned}
$$

where $Z_{c}=\frac{\rho c}{S}$ is the characteristic impedance for plane waves inside the resonator of cross section $S, \rho$ is the air density and $c$ is the sound velocity. The notations used for the dimensionless quantities are taken from the book [20]. Likewise, it is convenient to define a dimensionless reed opening parameter:

$$
\zeta=Z_{c} W H \sqrt{\frac{2}{\rho p_{M}}}
$$


where $W$ is the width of the reed. The two dimensionless parameters controlled by the player for a given fingering are $\gamma$ and $\zeta$, i.e., the blowing pressure and the reed opening parameter respectively.

Mainly based on considerations from Kergomard [21], an explicit expression for the air flow $u$ is given below:

$$
\begin{array}{ll}
u=\zeta(1-\gamma+p) \sqrt{|\gamma-p|} \operatorname{sgn}(\gamma-p) & \text { if } \gamma-p \leq 1 \\
u=0 & \text { if } \gamma-p \geq 1
\end{array}
$$

The first equation corresponds to the case of an open reed channel. In that case, the incoming air flow depends only on the pressure drop $\gamma-p(t)$ between the mouth and the mouthpiece. When the tip of the reed gets in contact with the lay, it completely closes the reed channel, therefore canceling the air flow, which is expressed by the second equation. This is another simplification since experiments with blowing machine [22] show that the reed deformation does not perfectly fit to the profile of the lay of the mouthpiece, inducing residual air flow.

The reduced input impedance of the instrument, denoted $Z(\omega)$, is defined in the frequency domain as the ratio between the pressure $P(\omega)$ and the air flow $U(\omega)$ into the mouthpiece. It can be written as the modal decomposition:

$$
Z(\omega)=\mathrm{j} \omega \sum_{n} \frac{F_{n}}{\omega_{n}^{2}-\omega^{2}+\mathrm{j} \omega \omega_{n} / Q_{n}},
$$

where $\omega_{n}$ is the pulsation, $Q_{n}$ is the quality factor and $F_{n}$ is the modal factor of the $n$th resonance. Simplification is obtained by truncating the series. To obtain the simplest model of single-reed instruments, the series (7) can be truncated at first order $(n=1)$ [23]. However in order to obtain quasi-periodic oscillations, an additional degree of freedom must be added [24]. The series (7) is therefore truncated at the second order $(n=2)$. The inverse Fourier transform of the truncated series allows one to model a reed instrument as a self-sustained oscillator defined by the following coupled system:

$$
\left\{\begin{array}{l}
\frac{\mathrm{d}^{2}}{\mathrm{~d} t^{2}} p_{1}(t)+\frac{\omega_{1}}{Q_{1}} \frac{\mathrm{d}}{\mathrm{d} t} p_{1}(t)+\omega_{1}^{2} p_{1}(t)=F_{1} \frac{\mathrm{d}}{\mathrm{d} t} u(t) \\
\frac{\mathrm{d}^{2}}{\mathrm{~d} t^{2}} p_{2}(t)+\frac{\omega_{2}}{Q_{2}} \frac{\mathrm{d}}{\mathrm{d} t} p_{2}(t)+\omega_{2}^{2} p_{2}(t)=F_{2} \frac{\mathrm{d}}{\mathrm{d} t} u(t) .
\end{array}\right.
$$

The pressure inside the mouthpiece $p(t)$ is defined as the sum of the two components $p_{1}(t)$ and $p_{2}(t)$.

The modal parameters $\omega_{n}, Q_{n}$ and $F_{n}$ are fitted to the digitization of the modulus of the input impedance measured on an alto saxophone (extracted from article [13]) for the $\mathrm{G}$ fingering on the first register, denoted $G^{\prime}$ thereafter (see Figure 2). Table 1 shows the values of the modal parameters resulting from the fitting and used in the remainder of this article. For this fingering, the first two peaks of the impedance curve have a much greater amplitude than the other peaks (see Figure 17 in [13]). The truncation of series (7) to order two is then considered as sufficient in this case. 


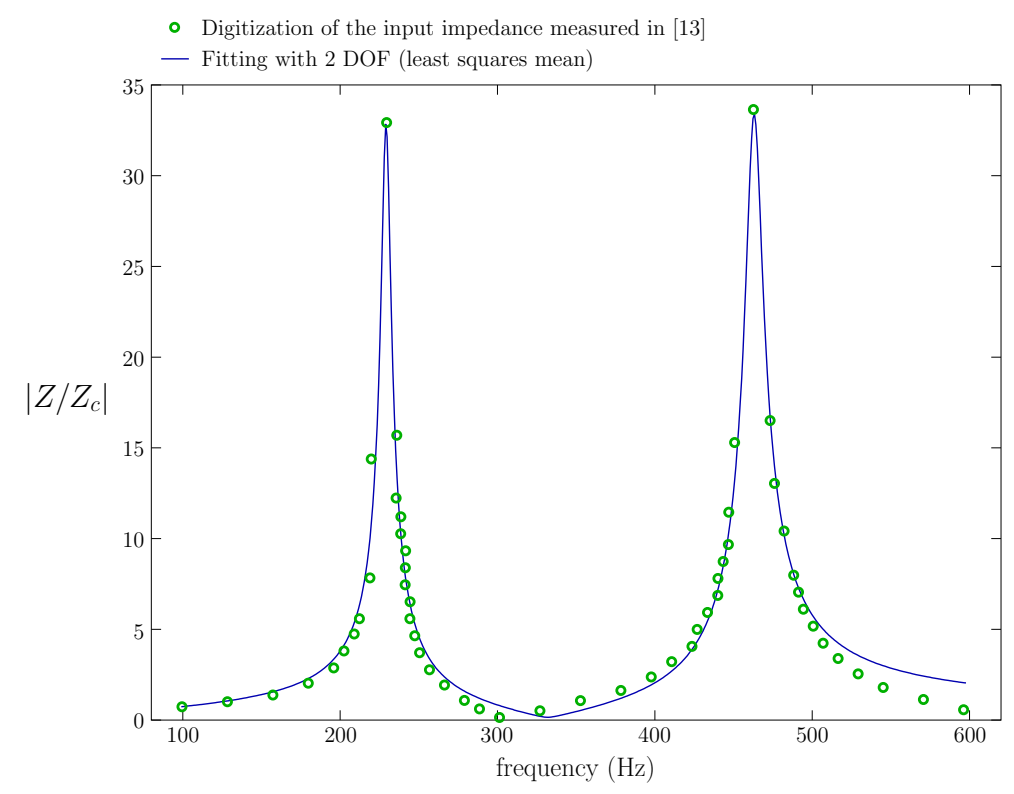

Fig. 2: Modulus of the reduced input impedance of an alto saxophone (fingering $G^{\prime}$ ): digitization of the impedance measured in [13] (circles) and modal fit with two modes (plain line).

\begin{tabular}{|l|c|c|c|}
\cline { 2 - 4 } \multicolumn{1}{c|}{} & $\omega_{n}$ & $F_{n}$ & $Q_{n}$ \\
\hline 1st mode & 1440 & 1322 & 36.6 \\
2nd mode & 2903 & 2386 & 41.2 \\
\hline
\end{tabular}

Table 1: Values of the modal parameters for the input impedance of an alto saxophone (fingering $G^{\prime}$ ). 


\subsection{Numerical solution of the non-linear system}

The system of equations (8) is solved using an ordinary differential equation solver. For this purpose, the second-order system (8) is recast into a couple of first order ordinary differential equations as follows:

$$
\left\{\begin{array}{l}
\frac{\mathrm{d}}{\mathrm{d} t} p_{1}=P_{1}(t) \\
\frac{\mathrm{d}}{\mathrm{d} t} P_{1}(t)=F_{1} \frac{\mathrm{d}}{\mathrm{d} t} u(t)-\frac{\omega_{1}}{Q_{1}} P_{1}(t)-\omega_{1}^{2} p_{1}(t) \\
\frac{\mathrm{d}}{\mathrm{d} t} p_{2}=P_{2}(t) \\
\frac{\mathrm{d}}{\mathrm{d} t} P_{2}(t)=F_{2} \frac{\mathrm{d}}{\mathrm{d} t} u(t)-\frac{\omega_{2}}{Q_{2}} P_{2}(t)-\omega_{2}^{2} p_{2}(t)
\end{array}\right.
$$

As multiple time scales are involved in the problem (duration of blowing pressure transient, bore resonance frequencies) and since the equations are not the same when the reed channel is closed or open, an ODE solver designed for stiff problems, namely ode23s from the Matlab ODE Suite is used. It is based on a modified Rosenbrock method [25]. As shown in the article [26], the use of this kind of commercial solvers is suitable for modeling wind instruments.

Using a solver requires the setting of initial states of the dynamical system (9), i.e. initial conditions for variables $p_{1}, p_{2}, P_{1}=\mathrm{d} p_{1} / \mathrm{d} t$ and $P_{2}=\mathrm{d} p_{2} / \mathrm{d} t$. The pressure inside the mouthpiece at the onset of sound production is still not well understood. Therefore, the initial conditions used for the resolution of the model are chosen arbitrarily. A pressure is imposed at time $t=0$ on the first mode of the bore, which is represented by the condition $p_{1}(t=0)=0.01$. This low value corresponds to a numerical perturbation, which in practice is sufficient to let the self-oscillation start. The other initial conditions are set to zero. This question would deserve further research since different initial conditions may lead to different steady state regimes, as shown in the case of periodic regimes [27].

\section{Classification of oscillation regimes}

The dynamical system (8) being nonlinear, it is very difficult to guess the influence of either control parameters (blowing pressure $\gamma$, reed opening parameter $\zeta$ ) or instrument design parameters (modal parameters of the two modes) on the nature of the oscillation regimes. To predict parameter values that can get rise to periodic regimes, the numerical continuation of solution branches [28-30] can be used. However, only static or periodic solution branches are tractable [31,32] and studying the influence of more than one parameter through numerical continuation (codimension of the bifurcation larger than 1) has never been done on models of musical instruments.

To the authors' knowledge, this kind of approach cannot be used to determine the influence of model parameters on the ability to produce quasi-periodic oscillations. This is the reason why we choose to sample the space of model parameters and to examine the resulting numerical solutions. As shown in a recent work [27], a Support Vector Machine (SVM) classifier is well suited for defining explicit boundary between different regimes of a similar model in the parameter space. This classification method is presented in Section 3.2. The criterion used to identify the nature of the oscillation regime is presented below. 


\subsection{Criterion to identify quasi-periodic regimes}

Quasi-periodicity is detected by studying the mouthpiece pressure signal. More specifically, the discriminating quantity is the power envelope of the mouthpiece pressure signal, denoted $p_{e}$. This quantity is estimated with the toolbox YIN [33], which is based on autocorrelation method. Figure 3 compares this quantity for periodic and quasi-periodic signals obtained from the numerical solution of the model. As expected, in the stationary part, the modulation of the instantaneous power is larger for the quasi-periodic signal. These differences can be synthesized with the following quantity:

$$
\epsilon=\frac{\operatorname{Var}\left(p_{e}\right)}{<p_{e}>}
$$

where $\operatorname{Var}\left(p_{e}\right)$ and $\left\langle p_{e}>\right.$ are the variance and the mean of signal $p_{e}$ respectively. The quantity $\epsilon$ is calculated from a stationary part of the pressure signal (see Figure 3).

This indicator being non strictly equal to zero for a periodic signal, a threshold must be estimated in order to distinguish between periodic and quasi-periodic regimes. Hence, the descriptor $\epsilon$ is calculated on a regular grid in the space of control parameters $(\gamma, \zeta)$. For each combination of $(\gamma, \zeta)$, system (8) is solved numerically thus providing the corresponding $\epsilon$ values. Results for 450 combinations of the parameters $\gamma$ and $\zeta$ are plotted in Figure 4 . The inharmonicity is defined as a relative difference between the first two resonance frequencies $\Delta f / f$. In this study, the inharmonicity is set to $150 \%$. This is implemented by replacing the pulsation $\omega_{1}$ by the value $\omega_{2} / 2.5$ in the parameters given in Table 1.

The set of points is comprised of two subsets, which can be separated by the horizontal plane $\epsilon=\epsilon_{\mathrm{th}}$. The precise value of $\epsilon_{\mathrm{th}}$ is not critical since the two subsets appear clearly separated. Therefore, the classification is expected to be robust with respect to the choice of $\epsilon_{\mathrm{th}}$. The value $\epsilon_{\mathrm{th}}=1.10^{-2} \%$ is chosen by visual inspection. This threshold has been tested to remain valid for other inharmonicity values between $100 \%$ to $200 \%$. Therefore, Figure 4 would be similar for a different inharmonicity value.

The dots above the plane $\epsilon=\epsilon_{\text {th }}$ are colored in black (dark blue in colored version), while those below this plane are colored in light grey (orange in colored version). The analysis of pressure signals corresponding to black dots reveals that quasi-periodic oscillations are characterized by a spectrum built around two incommensurate frequencies $f_{1}$ and $f_{2}$ as shown in Figure 5(a). Similarly, light grey dots (orange in color version) correspond to periodic oscillations characterized by a spectrum built around a fundamental frequency, as shown in Figure 5(b).

Therefore, the following criterion is retained: $-\epsilon<1.10^{-2} \%$ the signal is classified as "periodic"; $-\epsilon \geqslant 1.10^{-2} \%$ the signal is classified as "quasi-periodic".

\subsection{SVM classification}

The basic idea is to construct the boundaries of an $n$-dimensional map using a Support Vector Machine (SVM) [17,18], which provides an explicit expression of the boundary in terms of the chosen parameters. SVM is a machine learning technique that is widely used for classification. In optimization and reliability assessment, SVM is used to approximate highly nonlinear constraints and limit-state functions. The most important features of SVMs are their ability to handle multiple criteria using a single classifier, to be insensitive to discontinuities [34], and to be computationally very efficient. The ability of an SVM to 


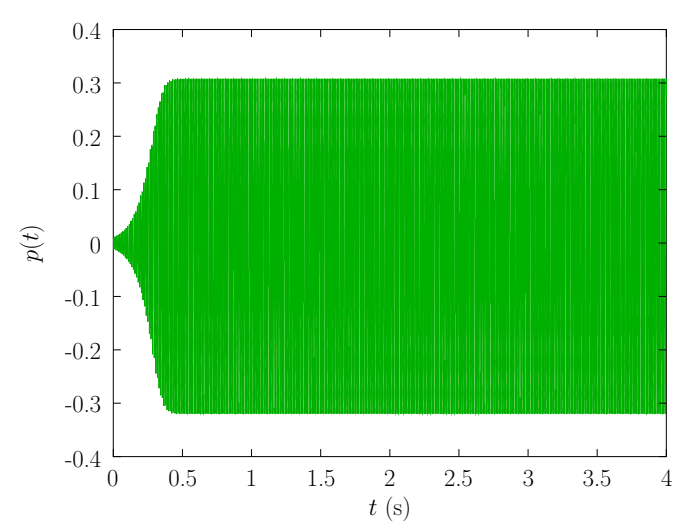

(a) Example of a periodic signal given by the model $(\gamma=0.7$ and $\zeta=0.4)$

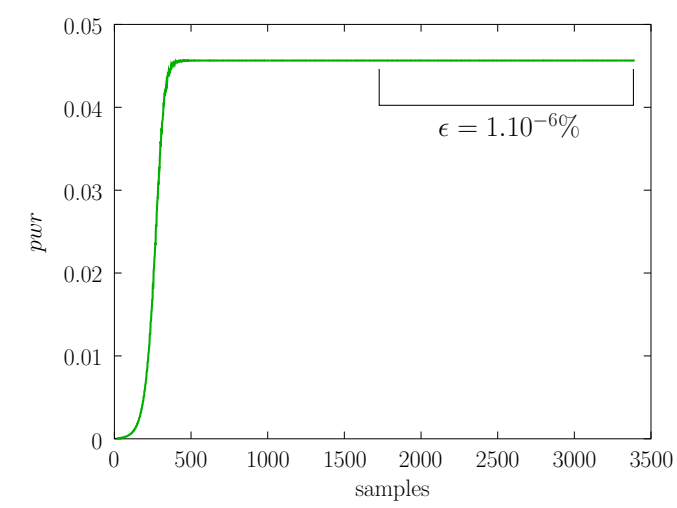

(c) Evolution of the instantaneous power of the periodic signal

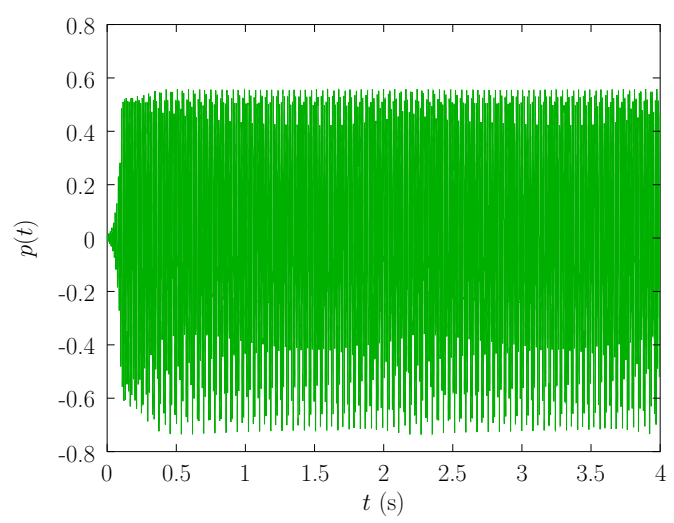

(b) Example of quasi-periodic signal given by the model $(\gamma=0.5$ and $\zeta=0.3)$

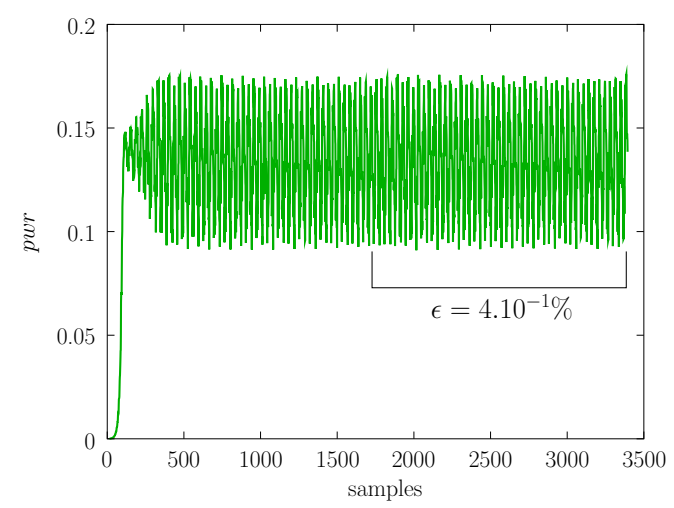

(d) Evolution of the instantaneous power of the quasi-periodic signal

Fig. 3: Comparison between the instantaneous power of a periodic and a quasi-periodic signal. The descriptor $\epsilon$, defined by Eq. (10), corresponds to the relative power envelope variance during a stable portion of the signal.

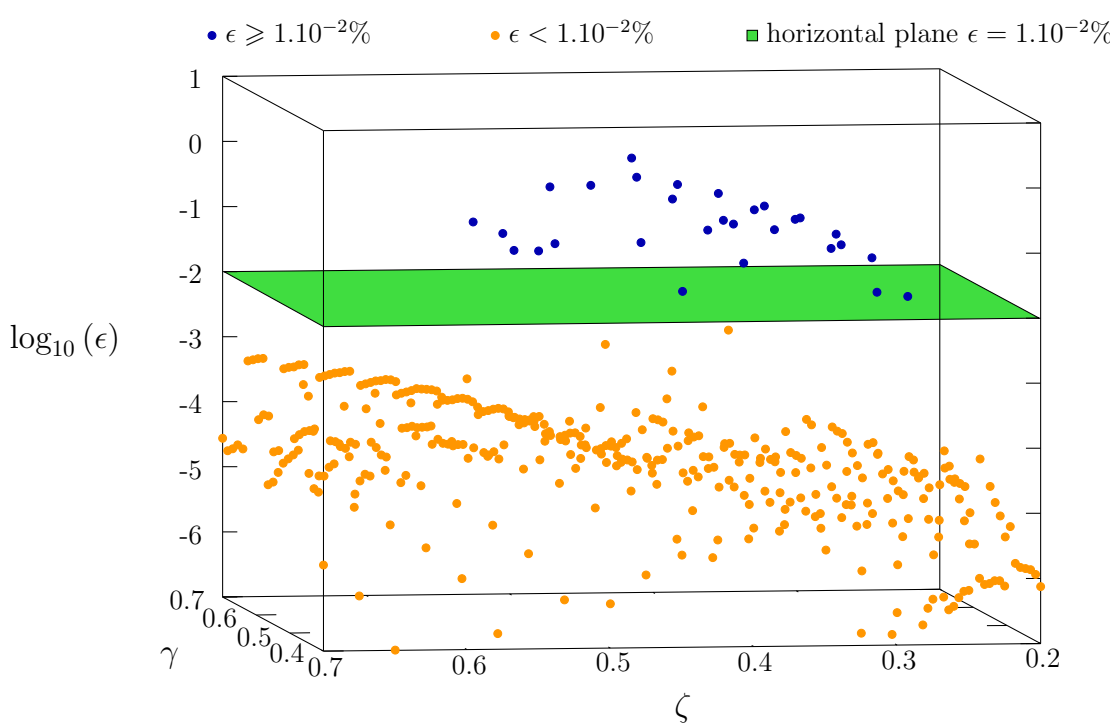

Fig. 4: Descriptor $\epsilon$ (see Eq. (10)) calculated on the mouthpiece pressure signal for a regular mesh of the control parameters space (inharmonicity is set to $\Delta f / f=150 \%$ and $\epsilon$ is expressed as a percentage). 


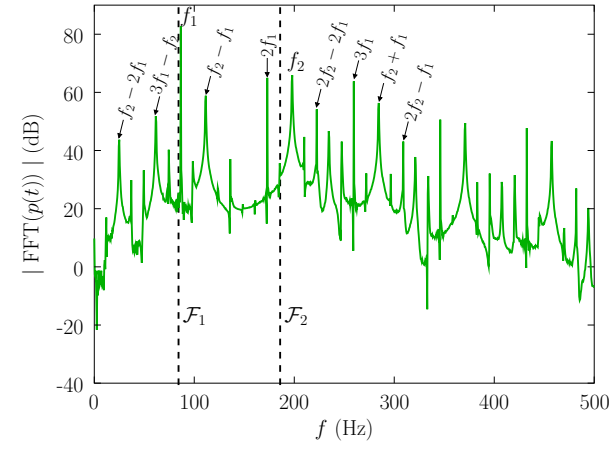

(a) Spectrum of a quasi-periodic signal whose descriptor $\epsilon$ is equal to $4.10^{-1} \%$ (black/blue dot from Figure 4). $f_{1}$ and $f_{2}$ are two incommensurate frequencies whose linear combinations correspond to the peaks frequencies in the spectrum.

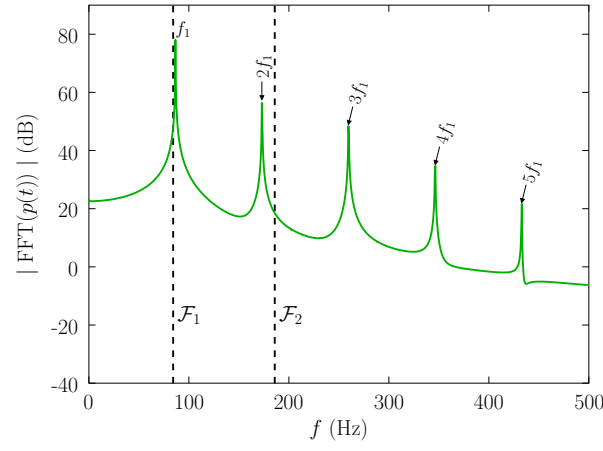

(b) Spectrum of a periodic signal whose descriptor $\epsilon$ is equal to $1.10^{-6} \%$ (light grey/orange dot from Figure 4).

Fig. 5: Spectra of periodic and quasi-periodic mouthpiece signals shown in Figure $3\left(\mathcal{F}_{1}\right.$ and $\mathcal{F}_{2}$ are the two resonance frequencies of the resonator).

handle discontinuities is essential in the case of sudden changes in the nature of oscillation regimes.

An SVM defines an explicit boundary that separates samples belonging to the two classes: periodic regime $\left(\epsilon<1.10^{-2 \%}\right)$ and quasi-periodic regime $\left(\epsilon \geqslant 1.10^{-2 \%}\right)$, characterized respectively by the values $y_{i}=-1$ and $y_{i}=1$, which are referred to as class labels. The $n$-dimensional vectors $\mathbf{x}_{i}$ are the training samples in the parameter space. In this paper, the selected parameters are: the blowing pressure $\gamma$, the reed opening parameter $\zeta$, and the inharmonicity $\Delta f / f$ therefore the vector $\mathbf{x}_{i}$ is written: $\left\{\gamma_{i}, \zeta_{i},\left.\frac{\Delta f}{f}\right|_{i}\right\}$. Given a set of $N$ training samples, an SVM boundary is given as the solution [17] of:

$$
s(\mathbf{x})=b+\sum_{i=1}^{N} \lambda_{i} y_{i} K\left(\mathbf{x}_{\mathbf{i}}, \mathbf{x}\right)=0
$$

where $b$ is a scalar referred to as the bias, $\lambda_{i}$ are Lagrange multipliers obtained from the quadratic programming optimization problem used to construct the SVM, and $K$ is a kernel function. The classification of any arbitrary point $\mathbf{x}$ is given by the sign of $s(\mathbf{x})$. The training samples for which the Lagrange multipliers are non-zero are referred to as the support vectors. The kernel function $K$ in Equation (11) can have several forms, such as polynomial or Gaussian radial basis kernel. The Gaussian kernel is used in this article:

$$
K\left(\mathbf{x}_{i}, \mathbf{x}_{j}\right)=e^{\left(\Gamma|| \mathbf{x}_{i}-\mathbf{x}_{j} \|^{2}\right)},
$$

where $\Gamma$ is a parameter dictating the "flexibility" of the SVM. In our work, $\Gamma=500$ was selected to accommodate the complexity of the boundaries obtained.

An initial approximation of the map is obtained using a design of experiments (DOE) [3537] such as Latin Hypercube Sampling. These DOE techniques are tailored so as to provide information over the whole space using a reasonable number of samples in higher dimensions.

The initial approximation of the boundary using a DOE might not be accurate and needs to be refined while maintaining a reasonable number of resolution of the single-reed instrument model. This refinement is performed using an adaptive sampling scheme that 
- Periodic regime $\left(\epsilon<1.10^{-2 \%}\right)$

- Quasi-periodic regime $\left(\epsilon \geqslant 1.10^{-2} \%\right)$
- Adaptative samples

- Initial samples

- - - Adapted SVM

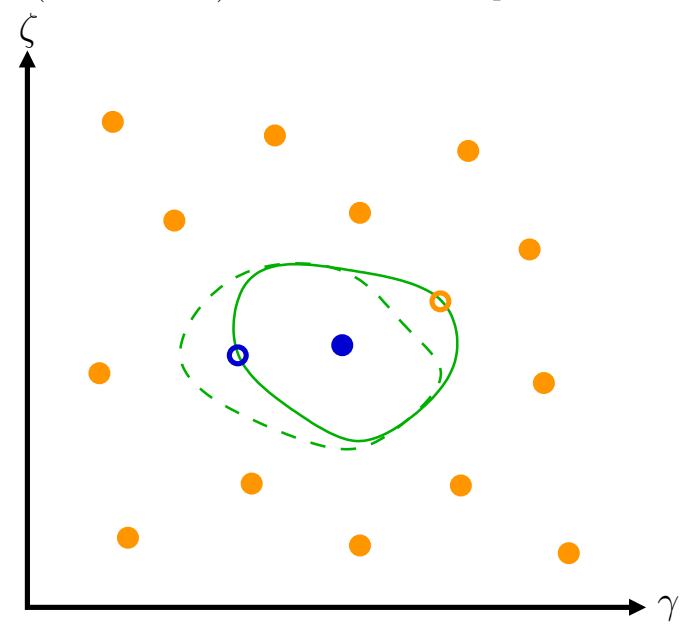

Initial SVM

Fig. 6: Schematic representation of the EDSD adaptive sampling scheme. The addition of samples (circles) in the sparse region of the space on the SVM is the basis of EDSD. The dashed boundary corresponds to the updated SVM.

is described in $[15,16]$. The basis of the scheme is to select a sample in the sparse regions of the space (i.e., as far away as possible from any existing sample) and also in the regions of highest probability of misclassification by the SVM. Formally, this amounts to solving the following optimization problem:

$$
\begin{array}{ll}
\max _{\mathbf{x}} & \left\|\mathbf{x}-\mathbf{x}_{\text {nearest }}\right\| \\
\text { s.t. } & s(\mathbf{x})=0
\end{array}
$$

with

$$
\mathbf{x}_{\min } \leq \mathbf{x} \leq \mathbf{x}_{\max }
$$

where $\mathbf{x}_{\text {nearest }}$ is the closest training sample (i.e., the one with minimum distance). The latter criterion is obtained by locating the samples on the SVM, hence the constraint $s(\mathbf{x})=0$ in Eq. (13). This represents a global (non-smooth) "max-min" optimization problem that can either be solved using a global optimizer such as a Genetic Algorithm or using a local optimizer with different starting points. A gradient-based technique such as Sequential Quadratic Programming can be used by reformulating the problem into a differentiable one as detailed in [15].

In order to illustrate the foundation of the algorithm, Figure 6 depicts an SVM constructed from two classes of samples (periodic and quasi-periodic samples) which is subsequently refined using an adaptive sample located in the most sparse region on the boundary.

\section{Experiments revisited numerically using the SVM classi- fication}

In this section, the experiments described in [13] are revisited using the numerical model presented in Section 2 and the classification method presented above, in order to check 
the ability of the model to reproduce qualitatively experimental trends. The following two sub-sections correspond to two different sets of experiments. First, the influence of the embouchure is studied for a fixed inharmonicity. In a second part, the characteristics of the produced sound are studied for different levels of inharmonicity. The first objective is to evaluate the ability of the model to reproduce similar trends as those described in the article [13]. The second objective is to identify the combinations of parameters that trigger quasi-periodicity.

\subsection{Change of control parameters}

\section{Description of the experiment carried out in [13]:}

Using a closed tube connected in parallel to the neck of an alto saxophone, the inharmonicity of resonance frequencies can be altered [13]. For a G' fingering and an inharmonicity fixed at $115 \%$ (corresponding to a decrease of 100 cents of the first resonance frequency), it is shown in [13] that modifying the lip pressure on the reed leads to either periodic or quasi-periodic oscillations (this is described from pages 46 to 47 in [13], in addition a video of this experience is viewable from the link given by the reference [38]).

\section{Numerical results}

To revisit this experiment with the model, a map is created following the EDSD approach detailed in Section 3.2, which permits to identify the various oscillation regimes in the plane $(\gamma, \zeta)$ that can be produced. The parameter $\zeta$ is directly linked to the pressure applied on the reed, since it includes the height of the reed channel at rest. The influence of the blowing pressure has not been studied in [13] but is included in our numerical study since it will prove to have a leading role in the triggering of quasi-periodicity.

Figure 7 highlights two different areas into the parameter space. The SVM boundary (solid line) separates periodic and quasi-periodic oscillation regimes localized in the lower left corner of the map. This map was obtained with 450 initial points, distributed throughout the parameter space whereas 250 adaptive samples were used to describe the complex shape of the boundary.

Figure 8 depicts the shape of the SVM according to the number of adaptive samples used. Despite the complex shape of the boundary, the first 50 adaptive points enable to get a relatively good estimate of the quasi-periodic region. Increasing the number of adaptive points allows progressively to refine the edges of the boundary: in this small area of the parameter space, more than 250 adaptive points are required. Such a density of samples could not have been generated over the entire parameter space, which demonstrates the need for adaptive sampling to detect this region of quasi-periodicity.

In order to check the consistency of the boundary, Figure 9 shows six time signals corresponding to six adaptive points, the location of which in the parameter space is indicated in Figure 8. These points were deliberately chosen near the border where the chance of misclassification is high. Nevertheless, none misclassification was detected. Indeed, Figures 9(a) and 9(f) show periodic signals (light grey/orange points on Figure 8). However, it is possible to see that their closeness to the region of quasi-periodicity has an effect during the transient part of the attack (slowly decaying oscillation of the pressure 


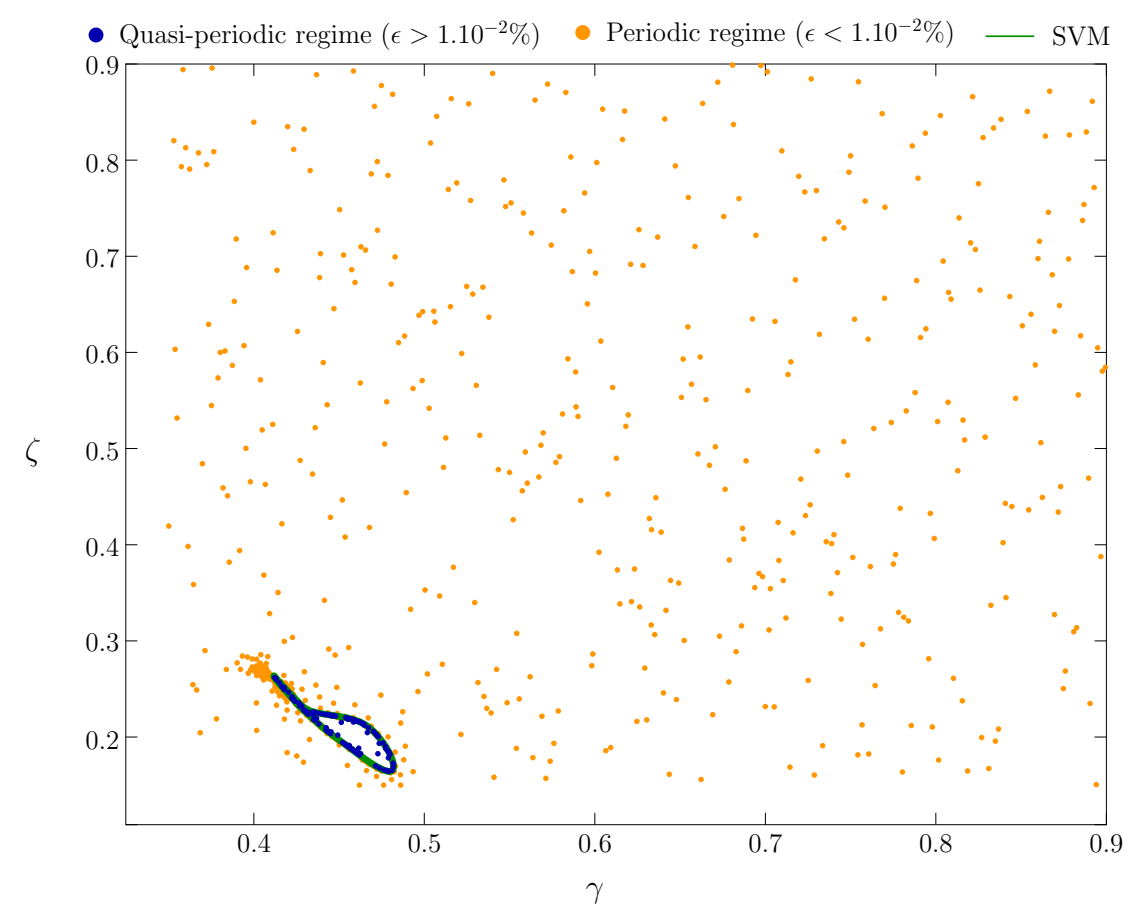

Fig. 7: Detection of quasi-periodic regimes using EDSD. The boundary between periodic and quasi-periodic regimes (solid line) is obtained using SVM with adaptive sampling (450 initial points and 250 adaptive points). The modal parameters of the resonator are given in Table 1 with $\omega_{1}=\omega_{2} / 2.15$.

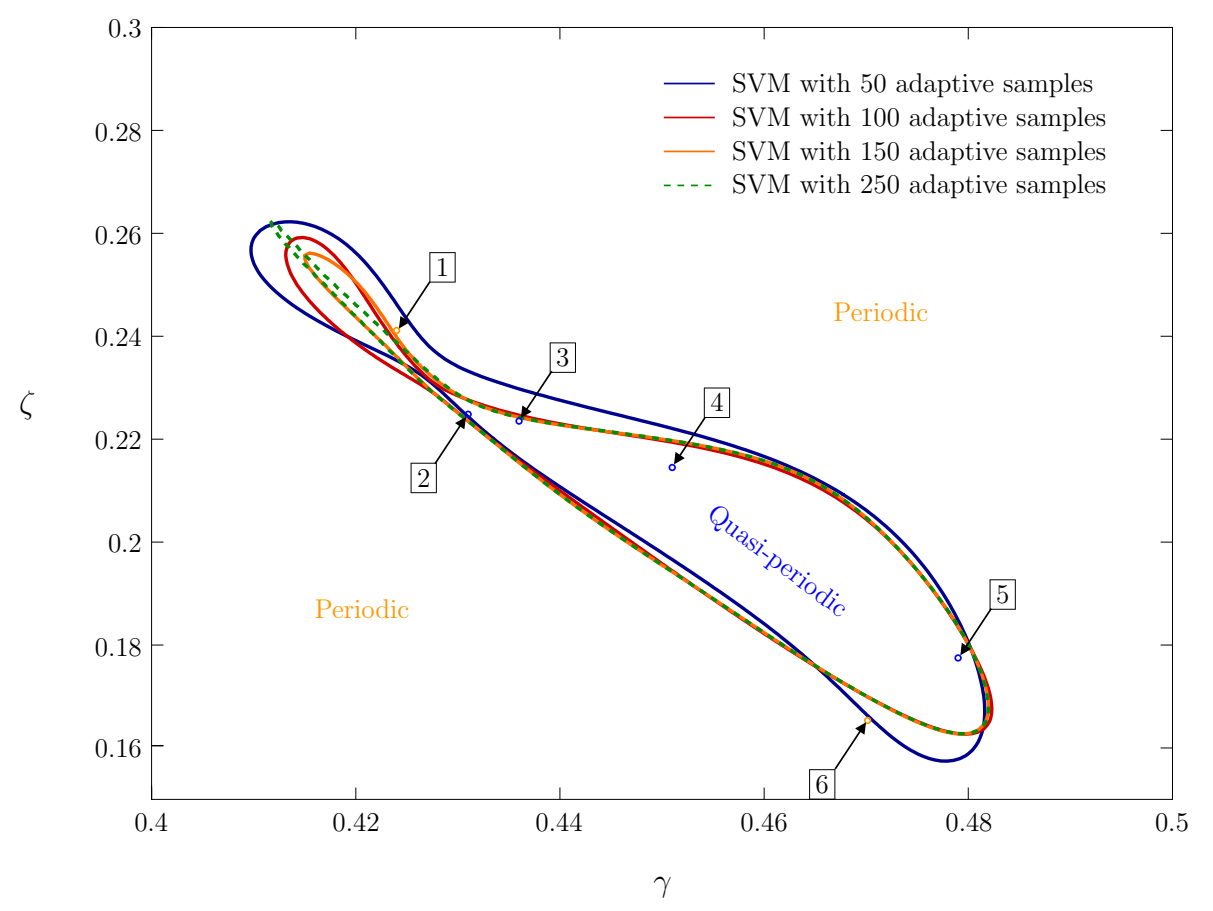

Fig. 8: Focus on the boundary between periodic and quasi-periodic regimes according to the number of adaptive points used. Samples have been omitted for sake of readability. The pressure signals represented by the points 1 to 6 are plotted on the Figure 9. 
envelope). Figures 9(b), 9(c), 9(d) and 9(e) depicts quasi-periodic signals (black/blue points on Fig. 8) comparable to those presented in the article [39]. It becomes obvious that the modulation frequencies can take different values depending on the control parameters. Generally, it can be seen on the time signals of Figure 9 that the transients are relatively long, which can result in a difficulty in the playability.

To better observe the relationship between duration of transients and quasi-periodicity, Figure 10 shows the transient duration for signals calculated in the vicinity of the quasiperiodic area (see Figure 8). Estimating the duration of transients is based on the time required to reach $80 \%$ of the maximum signal amplitude. Overall, the proximity of the bifurcation between static (no sound) and periodic regimes causes a progressive increase in the duration of the transient (from 0.1 to 0.35 seconds). Close to the boundary delimiting the periodic and quasi-periodic oscillations, some signals are characterized by a very long duration of transient. As shown in Figure 9(a), the amplitude modulations of the pressure signal appear in this area and explains the long duration of transients.

\section{Comparison with experiments from [13]}

The results presented above are in agreement with experimental observations given in reference [13]. The main agreement is that the embouchure setting has a determining influence on the existence of quasi-periodicity for a fixed inharmonicity. But in addition it is shown that the blowing pressure has a similar effect on the triggering of quasi-periodicity. This would deserve to be studied experimentally. Moreover, as observed experimentally in [13], modifying the lip pressure may have the effect of altering the modulation frequencies of quasi-periodic signals. As shown in Figure 9, not only a modification of the reed opening parameter $\zeta$ but also of the blowing pressure $\gamma$ allows the musician to choose the modulation frequency on a range from ten to one beat per second.

A third result suggested by both the experiment and the simulation, is that the inharmonicity may have an impact on the ease of playing. As it can be seen in Figure 9 and 10 , the duration of the attack transient significantly increases in the vicinity of the area of quasi-periodicity and the nature of the transient is altered as well, resulting in a quasi-periodic oscillation during the attack even for eventually periodic sounds (see Fig. 9(f)). More specifically, [13] makes the connection between the ease of playing and the occurrence of quasi-periodicity defined as "a limiting case of an unplayable note". This is explained by a possible increase of the inharmonicity caused by the dynamics of the reed (induced by a change of air volume inside the mouthpiece). In our case since the model does not take into account the dynamics of the reed, modifying the value of the mouth parameter $\zeta$ can not alter the inharmonicity. Our observation based on the duration of the transient of the dynamical system is therefore complementary to that proposed in [13].

\subsection{Variation of inharmonicity}

\section{Description of the experiment carried out in [13]:}

By a modification to the length of the piston connected in parallel to the neck, the first resonance frequency can be decreased by a maximum of 100 cents. Thereby, the inharmonicity of resonance frequencies can be continuously changed when the instrument is played [13], until the ratio $\Delta f / f$ reaches $115 \%$. During the progressive increase of the 


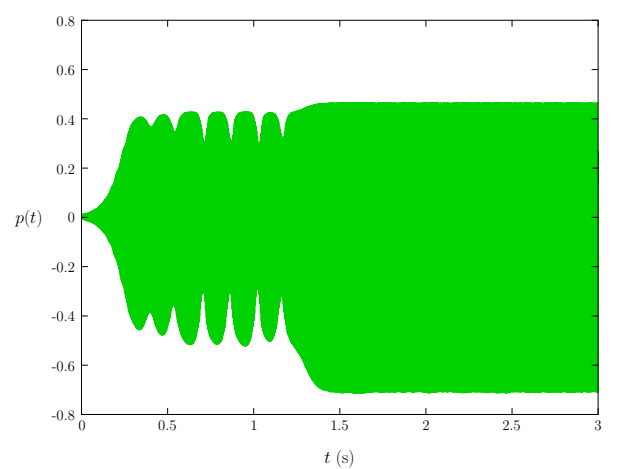

(a) Pressure signal for the light grey (orange in colored version) point 1 (see Fig. 8) $(\gamma=$ $0.424, \zeta=0.240)$

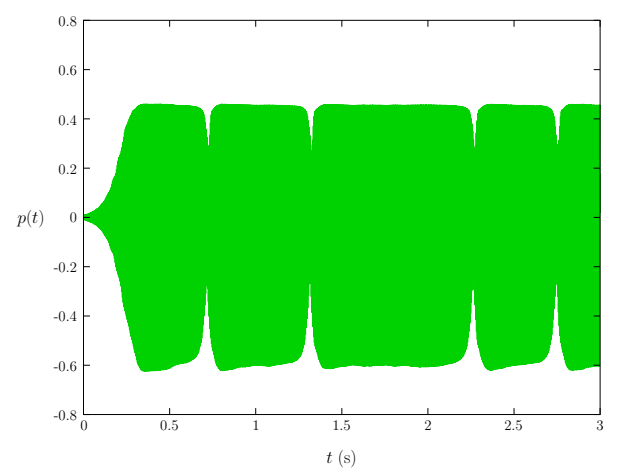

(c) Pressure signal for the black (dark blue in colored version) point 3 (see Fig. 8) $(\gamma=$ $0.436, \zeta=0.224)$

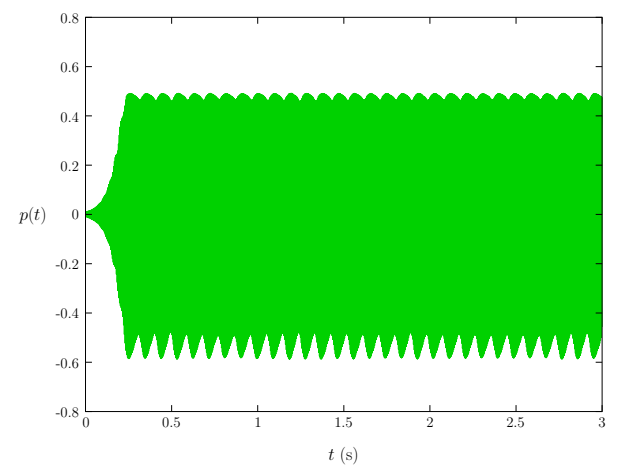

(e) Pressure signal for the black (dark blue in colored version) point 5 (see Fig. 8) $(\gamma=$ $0.479, \zeta=0.177)$

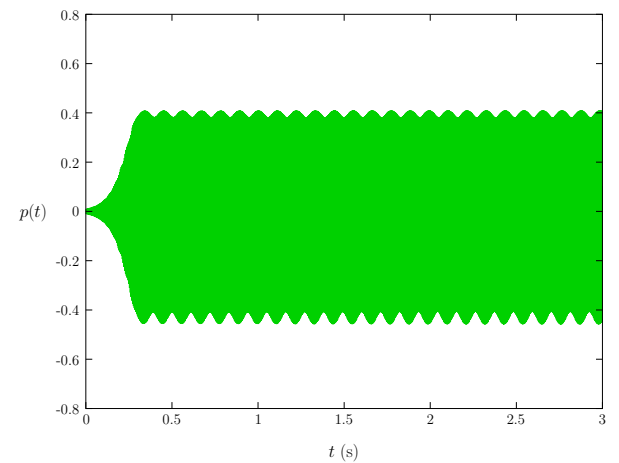

(b) Pressure signal for the black (dark blue in colored version) point 2 (see Fig. 8$)(\gamma=$ $0.431, \zeta=0.224)$

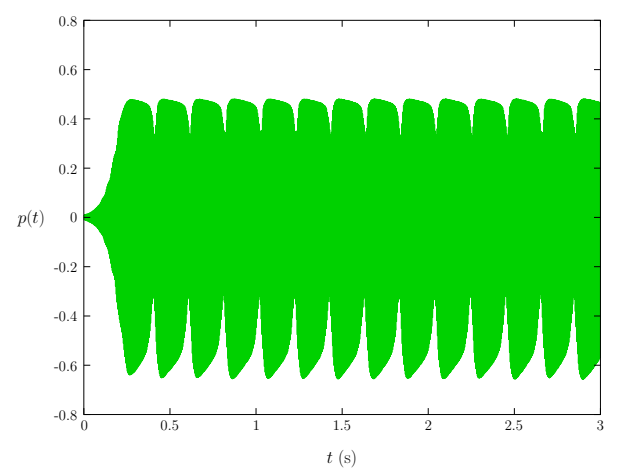

(d) Pressure signal for the black (dark blue in colored version) point 4 (see Fig. 8) $(\gamma=$ $0.451, \zeta=0.214$ )

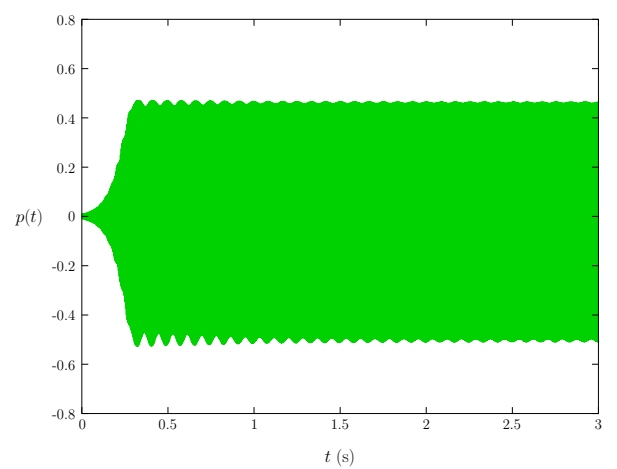

(f) Pressure signal for the light grey (orange in colored version) point 6 (see Fig. 8) $(\gamma=$ $0.470, \zeta=0.170$ )

Fig. 9: Comparison of pressure signals for periodic and quasi-periodic oscillation regimes, for combinations $(\gamma, \zeta)$ pinpointed in Figure 8. 


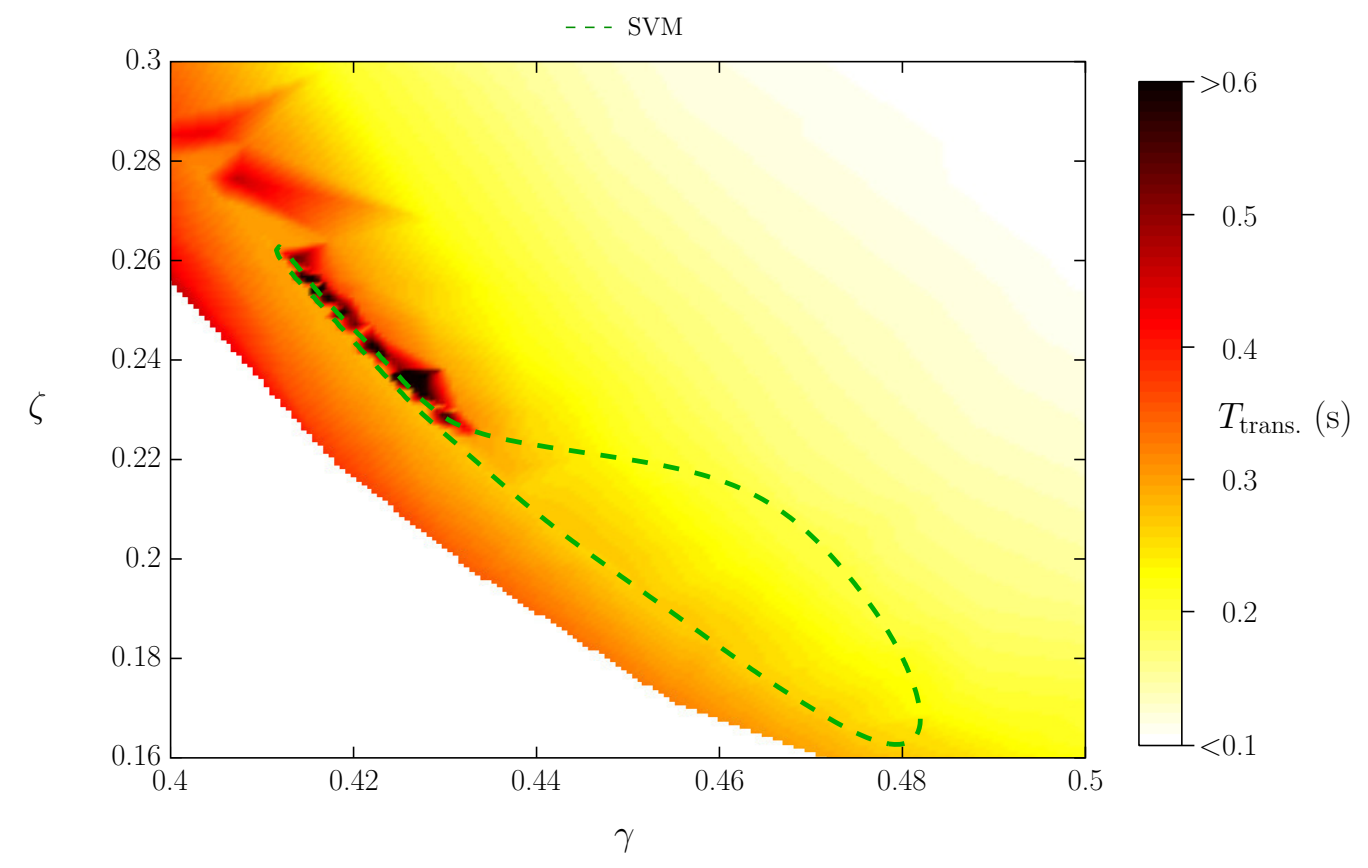

Fig. 10: Duration of transients in the parameter space. The duration of transients corresponds to the time required to reach $80 \%$ of the maximum signal amplitude.

inharmonicity, a bifurcation of the periodic regime occurs, leading to a quasi-periodic regime (a video of this experience is viewable from the link given by the reference [40].

\section{Numerical results}

In order to reproduce numerically this experiment, a 3D map is beforehand drawn so as to identify a couple of control parameters $\gamma$ and $\zeta$ that lead to quasi-periodic oscillations over a relatively wide range of inharmonicity. Figure 11 displays the boundary between the periodic and quasi-periodic regimes in the 3D space of parameters $\gamma, \zeta$ and $\Delta f / f$. This result has been obtained using the EDSD approach described in Section 3.2. The shape of the boundary predicted by the SVM appears as very complex. The general trend is that the more the inharmonicity increases, the more the area of quasi-periodicity becomes important in the parameter space. Moreover, it can be noticed that for an inharmonicity less than $110 \%$, no quasi-periodic regime is detected. On the other hand, for inharmonicity greater than $140 \%$, many areas of quasi-periodicity appear.

It is worth noting that this map was obtained with 500 initial points and 500 adaptive points, which is not much more compared to the previous two-dimensional map (Fig. 7). This underlines that the SVM method is particularly suitable for multidimensional classification.

Thanks to this map it is possible to choose a pair of control parameters for which the quasi-periodicity extends over a large range of inharmonicity $\Delta f / f$. The combination of $\gamma=0.47$ and $\zeta=0.28$ is used thereafter.

To study the dynamic behavior of the system (8), the inharmonicity is changed linearly over time in the simulation, while the control parameters $\zeta$ and $\gamma$ are kept constant. As described in the article [13], the modification of the inharmonicity is imposed by the 


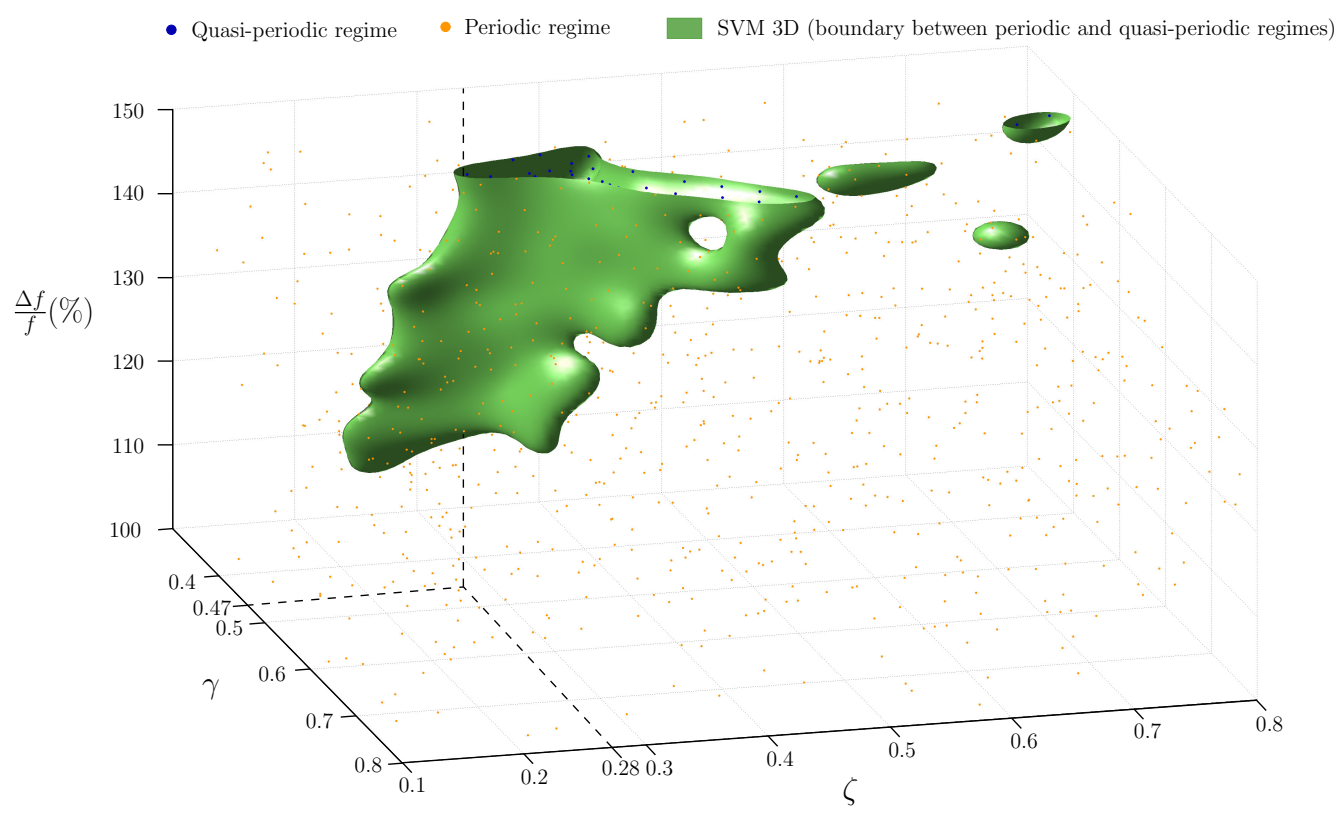

Fig. 11: Boundary delimiting the periodic and quasi-periodic regimes in the $3 D$ parameters space $\gamma, \zeta$ and $\Delta f / f$, obtained using the EDSD approach detailed in Section 3.2.

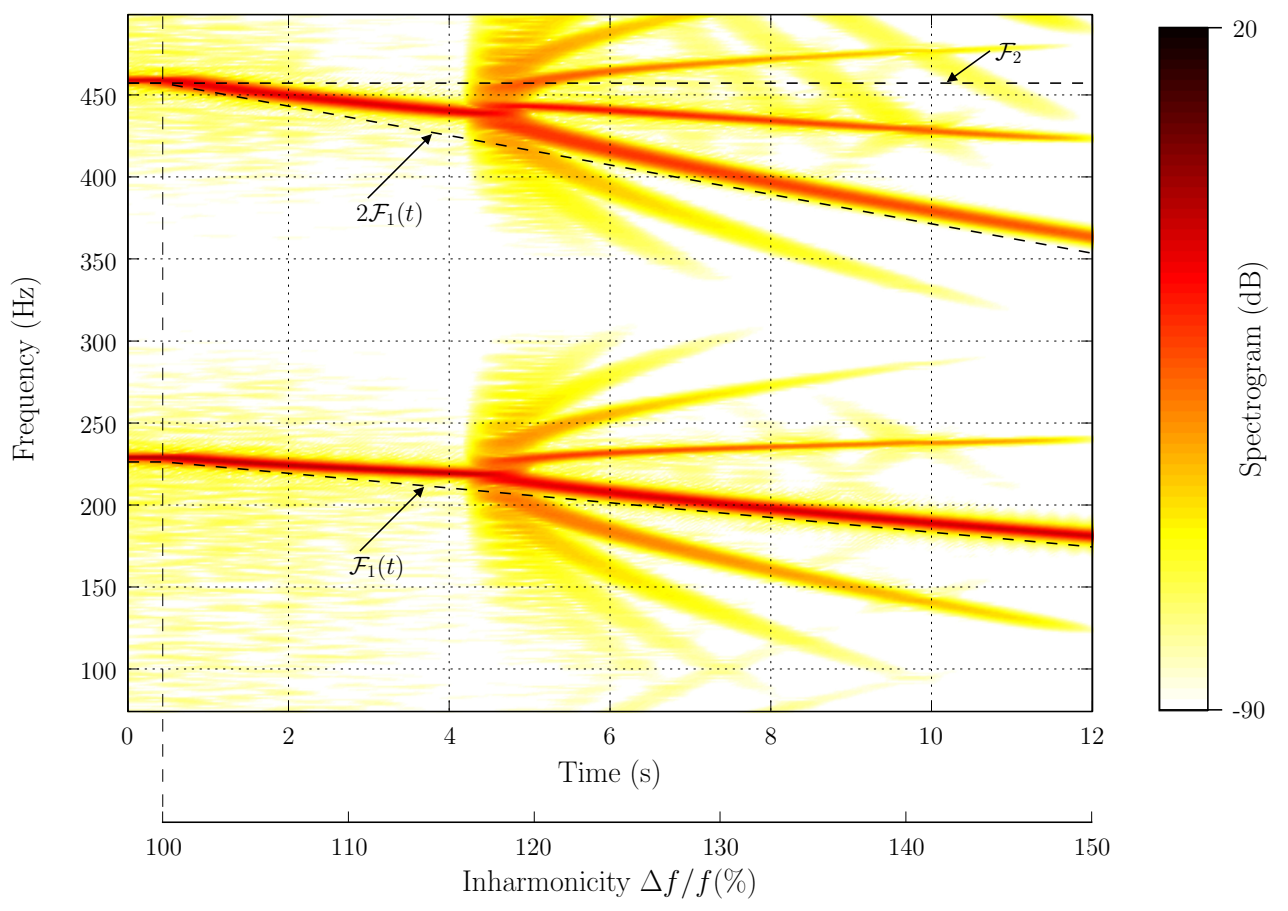

Fig. 12: Time-frequency representation of a pressure signal simulated for a variable inharmonicity over time. The inharmonicity increases linearly from the value $\Delta f / f=100 \%$ to $\Delta f / f=150 \%$. The control parameters are fixed: $\gamma=0.47$ and $\zeta=0.28 . \mathcal{F}_{1}$ and $\mathcal{F}_{2}$ are the first and second resonance frequencies respectively of the dynamical system (8). 
decreasing of the first resonance frequency, the second resonance frequency is constant. Figure 12 shows a time-frequency representation of the pressure signal thereby calculated. The inharmonicity is constant during the attack transient and varies linearly thereafter from the value $\Delta f / f=100 \%$ to $\Delta f / f=150 \%$. The maximum value $\Delta f / f=150 \%$ is imposed by the jump on the second register that occurs beyond this value.

One can notice that just after $t=4 \mathrm{~s}$, the dynamical system bifurcates to a quasiperiodic regime. This is characterized by the emergence of inharmonic components, combinations of two incommensurate frequencies. The outbreak of quasi-periodicity is effective for an inharmonicity equal to $117 \%$.

\section{Comparison with experiments from $[13]$}

The previous results are mainly in agreement with the experimental observations given by the article [13]. Figure 12 shows that the basic model considered in this paper is able to reproduce the three following trends observed experimentally [13]:

- The increase of the inharmonicity (i.e. the decrease of the first resonance frequency $\mathcal{F}_{1}$ ) causes a decrease of the fundamental frequency of the note G'. More precisely the decrease of the fundamental frequency is less than the decrease of $\mathcal{F}_{1}$.

- For a particular value of inharmonicity $(\Delta f / f=117 \%$ in the simulation), the oscillation becomes quasi-periodic.

- The quasi-periodicity is characterized by a set of linear combinations of two incommensurate frequencies.

However, concerning the latest point, the analysis of the simulation results reveals a difference with experimental results reported in [13]. Indeed page 46, Dalmont et al. explain that "it seems that the frequencies of the quasi-periodic oscillations are linear combinations of frequencies which are the resonance frequencies of the pipe in parallel with the reed". Figure 12 displays a different behavior for the model. Indeed, after analyzing, it appears that the two incommensurate frequencies making up the signal shown in Figure 12 do not correspond to the resonance frequencies of the instrument $\mathcal{F}_{1}$ and $\mathcal{F}_{2}$. However, it can be seen that the first resonance frequency is relatively close to one of the two signal components.

\subsection{Simulation with a C\#' fingering}

The results presented above have been obtained for the G' fingering. However, the article [13] also presents experiments with the C\#' fingering. With this particular fingering, no quasi-periodic regime is produced experimentally.

To evaluate the relevance of the model, the classification method is used for this fingering in the space parameters $(\gamma, \zeta)$. As previously, the modal parameters $\omega_{n}, Q_{n}$ and $F_{n}$ are fitted on the digitization of the modulus of the input impedance measured on alto saxophone (extracted from article [13]) for the $\mathrm{C} \#$ fingering of the first register. The inharmonicity is fixed at $115 \%$. By using 450 initial samples, the resulting map does not contain any quasi-periodic signal. For this fingering and for this inharmonicity, even if quasi-periodic regimes might be produced by the model, they would represent a very small area in the space of mouth parameters, making it highly unlikely to obtain them experimentally. 
Here again, the two-mode model considered in this paper proves to behave, at least qualitatively, like the experimental results shown in article [13]. The EDSD based method quickly leads to useful results.

\section{Conclusion}

A first conclusion is that the basic model considered in this paper is able to produce quasi-periodic sounds. Since the mass and the damping of the reed are ignored, and only two modes of the resonator are retained, it is probably a minimum model in terms of number of degrees of freedom capable of producing quasi-periodic sounds.

Classification through the EDSD approach proved to be an effective and efficient way of identifying a desired behavior, namely quasi-periodicity, in a multidimensional parameter space.

Experimental observations reported in [13] have been reproduced (at least qualitatively) using a simple model which is able to generate quasi-periodic signals in different ways. Key parameters that may destabilize periodic orbits through Hopf bifurcations to produce quasiperiodic regimes have been studied: the blowing pressure $(\gamma)$, the mouthpiece parameter $(\zeta)$ and the inharmonicity $(\Delta f / f)$. The influence of these parameters on the production of quasi-periodic regimes as well as their coupling has been studied through the construction of maps using EDSD.

Finally, this numerical study gives a valuable insight on the global behavior of the model and suggests some questions that might be addressed analytically. For example, is it possible to demonstrate that a minimal inharmonicity is required to trigger quasi-periodicity?

\section{A Relevance of the approximation on the reed dynamic}

The minimal model presented in Section 2 is constructed by ignoring the reed dynamics. This approximation directly affects the value of the bifurcations thresholds for the oscillation regimes. To highlight the relevance of this approximation, the simulation carried out in Part 4.1 is repeated with the minimal model (presented in Section 2) plus the reed dynamic taken into account. Therefore, the air flow expression now depends on the reed position (denoted $x$ ) and can be written as follows [20]:

$$
\begin{array}{ll}
u=\zeta(1+x-\gamma) \sqrt{|\gamma-p|} \operatorname{sgn}(\gamma-p) & \text { if } \gamma-p \leq 1 \\
u=0 & \text { if } \gamma-p \geq 1
\end{array}
$$

Also, the motion of the reed, induced by the acoustic oscillation in the mouthpiece, is given by:

$$
\frac{1}{\omega_{r}^{2}} \frac{\mathrm{d}^{2}}{\mathrm{~d} t^{2}} x+\frac{q_{r}}{\omega_{r}} \frac{\mathrm{d}}{\mathrm{d} t} x+x=p(t)
$$

where $\omega_{r}$ and $q_{r}$ represent respectively the pulsation and the damping factor of the first reed resonance. This equation, representing the dynamic behavior of the reed, is coupled with the system (8) for the calculation of the mouthpiece pressure signal.

Figure 13 shows a comparison between the minimal model with and without reed dynamics. For this comparison, the model parameters are the same as those used in Section 4. Concerning the frequency and damping factor of the reed resonance, they are 


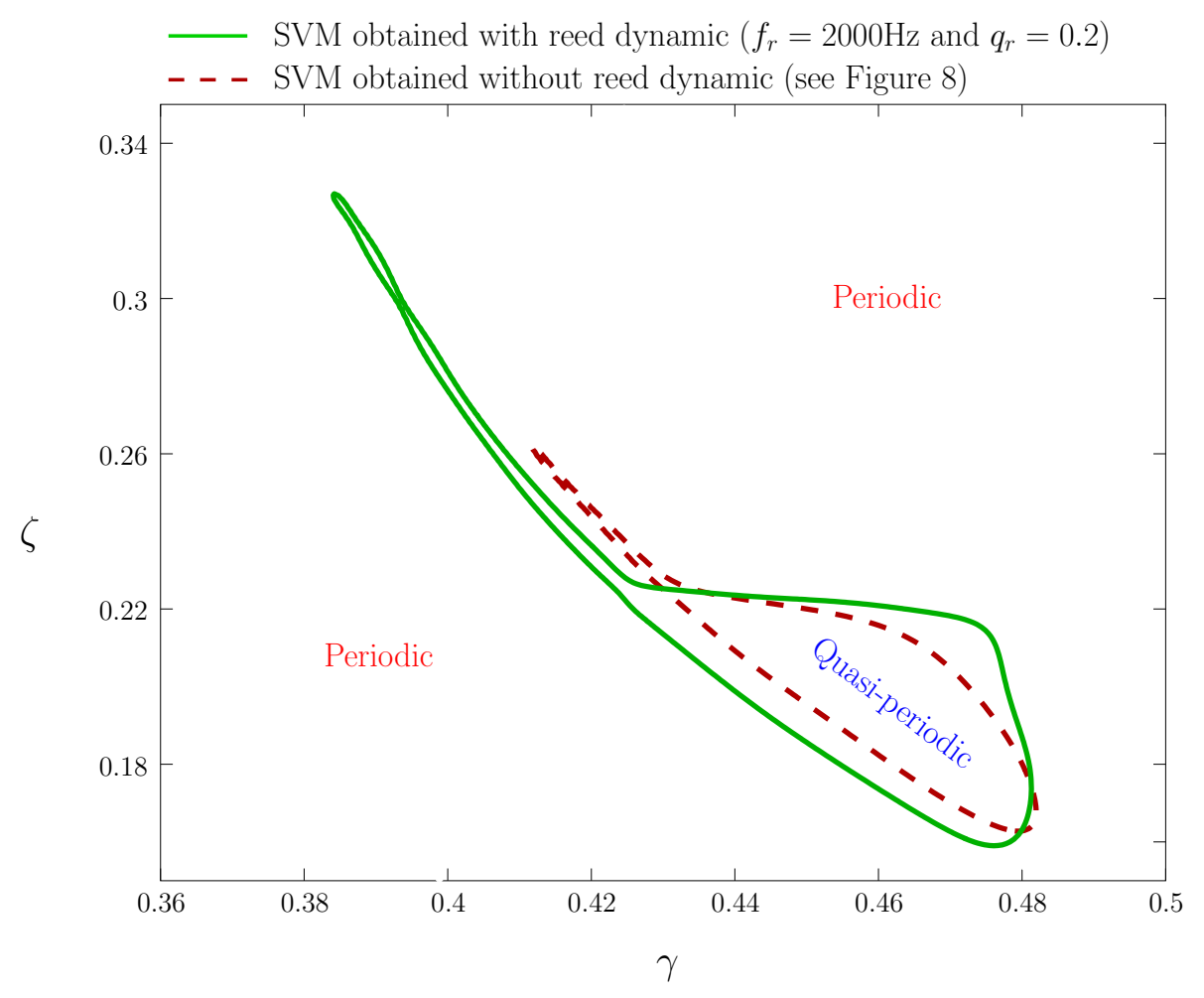

Fig. 13: Focus on the boundary between periodic and quasi-periodic regimes depending on the type of model used. The model parameters are the same as those used in Section 4.

fixed respectively to $f_{r}=2000 \mathrm{~Hz}$ and $q_{r}=0.2$. The order of magnitude of these values corresponds to that classically used for single reed instruments.

By comparison between the two boundaries shown in Figure 13, one can observe that the dynamics of the reed expands the area of quasi-periodicity. Also the trigger thresholds of quasi-periodic oscillations are slightly different. Within the framework of this study, the minimal model is intended only to describe the trends highlighted by the article [13]. In this case, it is thus relevant to ignore the dynamic behavior of the reed.

\section{Acknowledgments}

The authors are indebted to Joël Gilbert and Philippe Guillemain for precious technical discussions. Also, the authors wish to acknowledge Jean Kergomard for his valuable advices during the preparation of this manuscript. This work has been carried out in the framework of the Labex MEC (ANR-10-LABX-0092) and of the A*MIDEX project (ANR-11-IDEX-0001-02), funded by the «Investissements d'Avenir » French Government program managed by the French National Research Agency (ANR)

\section{References}

[1] N. H. Fletcher and H. D. Rossing. The physics of musical instruments. Springer-Verlag, 1998.

[2] A. H. Benade. Fundamentals of musical acoustics. Oxford University Press, 1976. 
[3] P Bergé, Y. Pomeau, and C. Vidal. Order within choas. Wiley, New York, 1984.

[4] V. Gibiat. Phase space representations of acoustical musical signals. Journal of Sound and Vibration, 123(3):529 - 536, 1988.

[5] W. Güth. Der wolfton beim cello. Acta Acustica united with Acustica, 41(3):163-176, 1978.

[6] C. J. Nederveen and J.-P. Dalmont. Pitch and level changes in organ pipes due to wall resonances. Journal of Sound and Vibration, 271(1-2):227 - 239, 2004.

[7] J. Backus. Multiphonic tones in the woodwind instruments. The Journal of the Acoustical Society of America, 63(2):591-599, 1978.

[8] N. H. Fletcher. Sound production by organ flue pipes. The Journal of the Acoustical Society of America, 60(4), 1976.

[9] N. H. Fletcher and L. M. Douglas. Harmonic generation in organ pipes, recorders, and flutes. The Journal of the Acoustical Society of America, 68(3), 1980.

[10] J. W. Coltman. Jet offset, harmonic content, and warble in the flute. The Journal of the Acoustical Society of America, 120(4):2312-2319, 2006.

[11] D. H. Keefe and B. Laden. Correlation dimension of woodwind multiphonic tones. The Journal of the Acoustical Society of America, 90(4):1754-1765, 1991.

[12] T. Idogawa, T. Kobata, K. Komuro, and M. Iwaki. Nonlinear vibrations in the air column of a clarinet artificially blown. The Journal of the Acoustical Society of America, 93(1):540-551, 1993.

[13] J.P. Dalmont, B. Gazengel, J. Gilbert, and J. Kergomard. Some aspects of tuning and clean intonation in reed instruments. Applied Acoustics, 46(1):19 - 60, 1995.

[14] F. Silva, V. Debut, J. Kergomard, C. Vergez, A. Deblevid, and P. Guillemain. Simulation of single reed instruments oscillations based on modal decomposition of bore and reed dynamics. In Proceedings of the International Congress of Acoustics, Sept 2007.

[15] A. Basudhar and S. Missoum. An improved adaptive sampling scheme for the construction of explicit boundaries. Structural and Multidisciplinary Optimization, pages $1-13,2010$.

[16] A. Basudhar and S. Missoum. A sampling-based approach for probabilistic design with random fields. Computer Methods in Applied Mechanics and Engineering, 198(4748):3647 - 3655, 2009.

[17] N. Cristianini and B. Schölkopf. Support vector machines and kernel methods: The new generation of learning machines. Artificial Intelligence Magazine, 23(3):31-41, 2002 .

[18] S.R. Gunn. Support vector machines for classification and regression. Technical Report ISIS-1-98, Department of Electronics and Computer Science, University of Southampton, 1998. 
[19] D. Noreland, S. Bellizzi, and C. Vergez. Nonlinear modes of clarinet-like musical instruments. Journal of Sound and Vibration, 324:983-1002, 2009.

[20] A. Chaigne and J. Kergomard. Acoustique des instruments de musique. Belin, 2008.

[21] J. Kergomard. Elementary Consideration on Reed-Instrument Oscillation. In G. Weinreich, A. Hirschberg, and J. Kergomard, editors, Mechanics of Musical Instruments, volume 335 of CISM Courses and Lectures, chapter 6. Springer-Verlag, Wien- New York, 1995.

[22] J.-P. Dalmont, J. Gilbert, and S. Ollivier. Nonlinear characteristics of single-reed instruments: Quasistatic volume flow and reed opening measurements. The Journal of the Acoustical Society of America, 114(4):2253-2262, 2003.

[23] V. Debut and J. Kergomard. Analysis of the self-sustained oscillations of a clarinet as a van der pol oscillator. In 18th International Congress on Acoustics-ICA, volume 2, pages 1425-1428, 2004.

[24] P. Bergé, Y. Pomeau, and C. Vidal. Order within chaos: towards a deterministic approach to turbulence. A Wiley-Interscience publication. Paris, 1986.

[25] Lawrence F. Shampine and Mark W. Reichelt. The matlab ode suite. SIAM J. Sci. Comput., 18(1):1-22, 1997.

[26] F. Silva, C. Vergez, Ph. Guillemain, J. Kergomard, and V. Debut. MoReeSC : a framework for the simulation and analysis of sound production in reed and brass instruments. Acta Acustica united with Acustica, 100:126-138, 2014.

[27] S. Missoum, C. Vergez, and J.-B. Doc. Explicit mapping of acoustic regimes for wind instruments, deposed in HAL (open access archive). http://hal . archives-ouvertes . fr/hal-00942597, 2014.

[28] S. Karkar, B. Cochelin, and C. Vergez. A high-order, purely frequency based harmonic balance formulation for continuation of periodic solutions: The case of non-polynomial nonlinearities. Journal of Sound and Vibration, 332(4):968-977, 2013.

[29] B. Cochelin and C. Vergez. A high-order, purely frequency based harmonic balance formulation for continuation of periodic solutions. Journal of Sound and Vibration, 324(1-2):243-262, 2009.

[30] E. J. Doedel and B. E. Oldeman. AUTO-07P : Continuation and Bifurcation Software for Ordinary Differential Equations. Concordia University, Montreal, Canada, 2009.

[31] S. Karkar, C. Vergez, and B. Cochelin. Oscillation threshold of a clarinet model: A numerical continuation approach. J. Acoust. Soc. Amer., 131(1):698-707, 2012.

[32] S. Terrien, C. Vergez, and B. Fabre. Flute-like musical instruments: A toy model investigated through numerical continuation. Journal of Sound and Vibration, 332(15):3833 $-3848,2013$.

[33] A. de Cheveigne and H. Kawahara. YIN, a fundamental frequency estimator for speech and music. J. Acoust. Soc. Am., 111(4):1917-1930, 2002. 
[34] S. Missoum, C. Dribusch, and P. Beran. Reliability-based design optimization of nonlinear aeroelasticity problems. Journal of Aircraft, 47(3):992-998, 2010.

[35] D.C. Montgomery. Design and Analysis of Experiments. Wiley and Sons, 2005.

[36] A.A. Giunta, S.F. Wojtkiewicz, and M.S. Eldred. Overview of modern design of experiments methods for computational simulations. In $41^{\text {st }}$ AIAA Aerospace Sciences Meeting and Exhibit, Reno, NV, 2003.

[37] J. Sacks, W. Welch, T. Mitchell, and H. Wynn. Design and analysis of computer experiments. Statistical Science, 4(4):409-423, Nov., 1989.

[38] J. Gilbert. Change of the control parameters on an inharmonic alto saxophone, (Video of the experiment). http://perso.univ-lemans.fr/ jgilbert/VideoSax/ SaxQdTbsurc.avi.

[39] B. Gazengel and J. Gilbert. From the measured input impedance to the synthesized pressure signal: application to the saxophone. In Proceedings of the International Symposium on Musical Acoustics, 1995.

[40] J. Gilbert. Change of the inharmonicity of an alto saxophone, (Video of the experiment). http://perso.univ-lemans.fr/ jgilbert/VideoSax/SaxQdTasurc .avi. 